\title{
Obesity-Related Adipokines Predict Patient-Reported Shoulder Pain
}

\author{
Rajiv Gandhi Anthony V. Perruccio Randy Rizek Omar Dessouki \\ Heather M.K. Evans Nizar N. Mahomed \\ Division of Orthopedic Surgery, University of Toronto, Toronto, ON, Canada
}

\section{Key Words}

Adipokines · Pain · Osteoarthritis · Shoulder

\begin{abstract}
Background/Aims: Increasingly, an inflammatory modulating effect of adipokines within synovial joints is being recognized. To date, there has been no work examining a potential association between the presence of adipokines in the shoulder and patient-reported outcomes. This study undertakes an investigation assessing these potential links. Methods: 50 osteoarthritis patients scheduled for shoulder surgery completed a pre-surgery questionnaire capturing demographic information including validated, patient-reported function (Disabilities of the Arm, Shoulder, and Hand questionnaire) and pain (Short Form McGill Pain Questionnaire) measures. Synovial fluid (SF) samples were analyzed for leptin, adiponectin, and resistin levels using Milliplex MAP assays. Linear regression modeling was used to assess the association between adipokine levels and patient-reported outcomes, adjusted for age, sex, BMI, and disease severity. Results: $54 \%$ of the cohort was female $(n=27)$. The mean age (SD) of the sample was 62.9 (9.9) years and the mean BMI (SD) was $28.1(5.4) \mathrm{kg} / \mathrm{m}^{2}$. From regression analyses, greater SF leptin and adiponectin levels, but not regarding resistin, were found to be associated with greater pain $(p<0.05)$. Adipokine levels were not associated with functional outcome scores. Conclusions: The identified association between shoulder-derived SF leptin and adiponectin and shoulder pain is likely explained by the pro-inflammatory characteristics of the adipokines and represents potentially important therapeutic targets.
\end{abstract}

(c) 2013 S. Karger GmbH, Freiburg

\section{Introduction}

The association between obesity and osteoarthritis $(\mathrm{OA})$ is believed to be a function of both local, mechanical factors and systemic biochemical factors [1-4]. The identification of an association between obesity and $\mathrm{OA}$ in non-weight-bearing joints, particularly the hands 
[5-7], has been a strong contributing factor to the latter view. Additionally, the current characterization of adipose tissue as an active endocrine organ which secretes pro- and antiinflammatory cytokines and adipokines (including leptin, adiponectin, and resistin) into the systemic circulation strengthens this view. This is due to strong evidence suggesting that changes in the balance of these modulating inflammatory factors can have net deleterious effects on cartilage turnover and repair mechanisms [8-12].

Studies have shown an association between serum and synovial fluid (SF) adipokine levels and knee pain $[13,14]$ as well as migraines [15], and one study showed that greater peritoneal leptin levels were associated with greater pelvic pain [16]. Three studies have identified an association between obesity and incident shoulder pain [17-19], and while a recent study noted a strong association between shoulder SF adipokine levels and body habitus [20], no study, to our knowledge, has assessed whether there is an association between shoulder SF adipokine levels and shoulder pain and function. The primary objective of our study was to investigate the association between shoulder SF leptin, adiponectin, and resistin levels and patient-reported shoulder pain and upper extremity function.

\section{Subjects and Methods}

OA patients awaiting shoulder surgery were recruited from a single, academic hospital between August 2009 and February 2011. Patients with a history of previous shoulder surgery, previous intra-articular steroid injection, posttraumatic arthritis, or a history of inflammatory arthropathy were excluded from the study. All patients were off narcotic/analgesic and non-steroidal anti-inflammatory medications at the time of questionnaire completion. The recruitment and consenting of patients was carried out by a study nurse not involved in the medical care of the patient. The study protocol was approved by the Human Subject Review Committee.

Prior to surgery, patients completed a questionnaire which elicited age, gender, comorbidity, and height and weight (used to calculate BMI $\left(\mathrm{kg} / \mathrm{m}^{2}\right)$ ) information. Additionally, the questionnaire included the Short Form McGill Pain Questionnaire (MPQ-SF) [21], to which patients were directed to respond in relation to their shoulder, and the Disabilities of the Arm, Shoulder, and Hand questionnaire (DASH) [22].

The MPQ-SF consists of 11 descriptors representing the sensory dimension of pain experience and 4 representing the affective dimension. Each descriptor is ranked on an intensity scale of ' $0=$ none' to ' $3=$ severe', with overall subscale scores ranging from 0 to 33 (sensory) and from 0 to 12 (affective). These two subscales (pain experience and affective dimension) can be combined into an overall score, and this was the outcome measure used in our study [21]. A higher score represents greater pain. The MPQ-SF has been validated for use across disease groups and surgical cohorts.

The DASH is a 30-item questionnaire designed to measure physical function and symptoms in people with any of several musculoskeletal disorders of the upper limb. The items assess the degree of difficulty when performing various physical activities (21 items), the severity of each of the symptoms of pain, activityrelated pain, tingling, weakness, and stiffness (5 items), and the degree of impact on social activities, work, and sleep (4 items). All items are scored on a scale ranging from 1 to 5 , and the overall score is scaled from 0 (no disability) to 100 (most severe disability).

Intraoperative assessment of the cartilage was performed by the treating surgeon according to the Outerbridge classification adapted from the knee [23]; grade 1 lesion: softening and swelling of the cartilage; grade 2 lesion: a partial-thickness defect with fissures on the surface that do not reach the subchondral bone or exceed $1.5 \mathrm{~cm}$ in diameter; grade 3 : loss of more than $50 \%$ of cartilage thickness; grade 4: complete loss of cartilage with subchondral bone exposure [23].

\section{Tissue Samples}

SF samples were collected from the patients at the time of shoulder surgery under sterile conditions. All patients were fasting 12-16 h prior to sample collection. Fluid samples were kept out of the light and stored in sterile containers at $-80^{\circ} \mathrm{C}$ until analyzed to minimize interassay variation. Samples were then centrifuged at 3,000 $\times g$ for $30 \mathrm{~min}$. Protease inhibitors were added immediately, and all samples were analyzed not more than 3 weeks after collection. Samples were analyzed for leptin, adiponectin, and resistin levels using the 
Gandhi et al.: Obesity-Related Adipokines Predict Patient-Reported Shoulder Pain

Table 1. Mean SF adipokine levels compared across MPQ-SF tertiles

\begin{tabular}{|c|c|c|c|}
\hline MPQ-SF & $\begin{array}{l}33 \text { rd percentile - } \\
5 \text { points }\end{array}$ & $\begin{array}{l}66 \text { th percentile - } \\
13 \text { points }\end{array}$ & $\begin{array}{l}\text { 99th percentile - } \\
25.5 \text { points }\end{array}$ \\
\hline Mean SF resistin pg/ml (SD) & $13,792.5(46,853.9)$ & $1,453.9(824.1)$ & $1,818.6(902.5)$ \\
\hline Mean SF leptin pg/ml (SD) & $1,836.9(2,291.2)$ & $2,294.1(3,683.6)$ & $3,436.9(6,382.4)$ \\
\hline Mean SF adiponectin $\mathrm{pg} / \mathrm{ml}$ (SD) & $1,570,552(1,184,135)$ & $2,060,130(1,665,980)$ & $2,767,793(1,788,611)$ \\
\hline
\end{tabular}

Table 2. Mean SF adipokine levels compared across DASH tertiles

\begin{tabular}{llll}
\hline DASH & $\begin{array}{l}\text { 33rd percentile - } \\
\text { 33 points }\end{array}$ & $\begin{array}{l}\text { 66th percentile - } \\
\text { 51 points }\end{array}$ & $\begin{array}{l}\text { 99th percentile - } \\
\text { 83 points }\end{array}$ \\
\hline Mean SF resistin pg/ml (SD) & $1,504.1(849.9)$ & $1,348.2(815.9)$ & $\begin{array}{l}17,579.7(52,735.9) \\
\text { Mean SF leptin pg/ml (SD) }\end{array}$ \\
$\begin{array}{ll}1,080.5(966.3) \\
\text { Mean SF adiponectin pg/ml (SD) }\end{array}$ & $2,086,933(1,644,380)$ & $2,128.7(3,675.1)$ & $4,861.5(6,838.6)$ \\
\hline
\end{tabular}

Milliplex MAP kits (Millipore). Concentrations were determined in triplicate for each sample to ensure accuracy. Our analysis for adiponectin recognizes all multimeric forms, but not the monomeric forms or the globular domain.

Performance characteristics for the immunoassays were determined with the intra-assay coefficient of variation (CV) for leptin and adiponectin individually. For the leptin assays, we found the following: intraassay CV SF $=11.34 \%$. For the adiponectin assays, we found the following: intra-assay CV SF $=1.89 \%$.

\section{Statistical Analysis}

Mean SF adipokine levels are shown across tertiles of MPQ-SF and DASH scores.

A linear regression model was assessed to investigate the association between our measures of interest: SF leptin, SF adiponectin, the adiponectin/leptin (A/L) ratio, and SF resistin levels for each of our dependent variables, i.e. MPQ-SF and DASH scores. The A/L ratio, which has been shown to be associated with patientreported knee OA pain [13], was calculated by dividing the adiponectin concentration by the leptin concentration for each patient. The models were adjusted for age, gender, BMI, and OA grade. Collinearity was assessed with tolerance statistics.

All statistical analyses were performed with SPSS Version 13.0 (Chicago, IL, USA). All reported p values are two-tailed with an alpha of 0.05 .

\section{Results}

Of the 50 study participants, 27 (54\%) were female. The mean age (SD) of the sample was 62.9 (9.9) years and the mean BMI was $28.1 \mathrm{~kg} / \mathrm{m}^{2}$ (range $21.8-46.7 \mathrm{~kg} / \mathrm{m}^{2}, \mathrm{SD} 5.4 \mathrm{~kg} / \mathrm{m}^{2}$ ). The prevalence of comorbidity was low at a mean of 0.2 per patient. The prevalence of hypertension was $16 / 50(32 \%)$ and $4 / 50(8 \%)$ for diabetes. The mean MPQ-SF and DASH scores were 10.8 (SD 8.6) and 43.2 points (SD 19.9), respectively. The mean (SD) SF adipokine levels were: leptin $2,489.5 \mathrm{pg} / \mathrm{ml}(4,403.9 \mathrm{pg} / \mathrm{ml})$; adiponectin 2,147,255 pg/ml (1,574,099 pg/ml); resistin $6,497.9 \mathrm{pg} / \mathrm{ml}(29,602.6 \mathrm{pg} / \mathrm{ml})$.

Mean SF adipokine levels across the tertiles of MPQ-SF and DASH scores are presented in tables 1 and 2, respectively. Across the MPQ-SF tertiles, a generally increasing concentration of leptin and adiponectin, but not of resistin, with increasing pain scores was found. The same was not observed across DASH score tertiles. 
Gandhi et al.: Obesity-Related Adipokines Predict Patient-Reported Shoulder Pain

Table 3. Linear regression modeling predicting MPQ-SF scores, adjusted for OA grade
Table 4. Linear regression modeling predicting DASH scores, adjusted for OA grade
Beta coefficient (95\% confidence p value interval) for predicting MPQ-SF

\begin{tabular}{lll}
\hline & $\begin{array}{l}\text { Beta coefficient (95\% confidence } \\
\text { interval) for predicting MPQ-SF }\end{array}$ & p value \\
\hline Age & $0.18(-0.25$ to 0.62$)$ & 0.40 \\
Female gender & $-2.43(-9.17$ to 4.30$)$ & 0.46 \\
BMI & $-0.57(-1.58$ to 0.44$)$ & 0.26 \\
SF leptin & $0.00141(0.0001-0.003)$ & 0.02 \\
SF adiponectin & $0.0000021(0.0000001-00001)$ & 0.049 \\
SF resistin & $0.000045(0.000002-0.0004)$ & 0.48 \\
\hline
\end{tabular}

\begin{tabular}{lll}
\hline & $\begin{array}{l}\text { Beta coefficient }(95 \% \text { confidence } \\
\text { interval) for predicting DASH }\end{array}$ & p value \\
\hline Age & $0.39(-0.75$ to 1.55$)$ & 0.48 \\
Female gender & $-9.6(-27.17$ to 8.05$)$ & 0.27 \\
BMI & $0.22(-2.40$ to 2.84$)$ & 0.86 \\
SF leptin & $0.00079(-0.002$ to 0.004$)$ & 0.59 \\
SF adiponectin & $0.0000047(0.0000002-00004)$ & 0.11 \\
SF resistin & $0.00013(0.00002-0.0005)$ & 0.42
\end{tabular}

Results from the linear regression modeling are presented in tables 3 and 4 for MPQ-SF and DASH outcome scores, respectively. Adjusted for age, sex, BMI, and OA grade, increasing concentrations of leptin and adiponectin were associated with increasing MPQ-SF pain scores. Resistin, however, was not found to be associated with pain scores. None of the SF adipokine levels were found to be associated with the DASH functional outcome score.

\section{Discussion}

Adipokines are known to regulate inflammation and have been shown to be associated with various types of pain [13-16]. In this study, we report that greater shoulder SF adiponectin and leptin concentration levels were found to be associated with greater MPQ-SF shoulder-specific pain scores among patients with shoulder OA. The same was not found, however, with DASH functional scores.

There is limited evidence linking SF adipokines and joint pain in OA. One group found that serum leptin levels were correlated with function scores but not pain among knee OA patients [24]. In previous work among knee OA patients, we found that the SF A/L ratio better predicted patient-reported pain than the individual adipokine levels alone [13]. This is in contrast to what we found in the present study. The reasons for these differences are not clear; however, this may further highlight the ongoing debate in the literature about the role of adiponectin in synovial joints, pro- or anti-inflammatory.

Leptin has been shown to be pro-inflammatory within synovial joints through the release

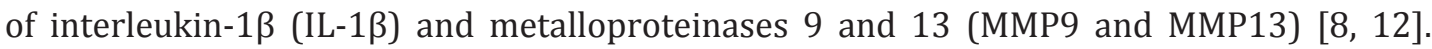
Further, it has been linked to neuropathic pain through its central action of up-regulating $\mathrm{N}$-methyl-D-aspartate receptors (NMDAR) [25]. The pain experience in OA has largely been divided into two categories: a dull aching pain that becomes constant over time; and a sharp, episodic, more emotionally debilitating pain (believed to be neuropathic) [26]. The MPQ-SF 
is used as a measure of neuropathic pain and also as an outcome for interventions for reducing neuropathic pain [27-29]. Our finding that leptin concentrations were associated with MPQ-SF pain scores but not with functional limitation (DASH) scores may perhaps strengthen the understanding of its role in the latter type of OA pain, rather than in the former [13].

The role of adiponectin within the joint has not been as clear. Studies have documented that adiponectin stimulates the release of anti-inflammatory factors such as IL-10 [30], tissue inhibitor of MMP2, and down-regulates IL-1 $\beta$-induced MMP13 levels [9]. However, there have recently been suggestions that adiponectin may have a more pro-inflammatory effect in synovial joints due to an increased production of nitric oxide synthase (NOS-2), MMP3, MMP9, and IL-8 [10, 31, 32]. Our findings that greater SF adiponectin concentration levels predicted greater pain scores possibly suggest a net pro-inflammatory role within the shoulder.

This study has its limitations. The study's cross-sectional nature precludes comment on whether the adipokines observed within the shoulder joint were antecedent or downstream (or both) to the prevalent OA and/or pain. Also, while the analyses were adjusted for a number of covariates, the possibility of unmeasured confounders exists. Finally, a control group of non-OA patients would be useful in future work.

Greater shoulder SF concentrations of leptin and adiponectin were associated with worse shoulder-specific pain scores among patients with shoulder OA. These findings suggest a likely pro-inflammatory role of leptin and adiponectin within the shoulder joint and points toward a potential target in the treatment of $\mathrm{OA}$ pain.

\section{Disclosure Statement}

No conflict of interest.

\section{References}

1 Felson DT, Anderson JJ, Naimark A, Walker AM, Meenan RF: Obesity and knee osteoarthritis. The Framingham study. Ann Intern Med 1998;109:18-24.

2 Bourne R, Mukhi S, Zhu N, Keresteci M, Marin M: Role of obesity on the risk for total hip or knee arthroplasty. Clin Orthop Relat Res 2007;465:185-188.

3 Pallu S, Francin PJ, Guillaume C, Gegout-Pottie P, Netter P, Mainard D, Terlain B, Presle N: Obesity affects the chondrocyte responsiveness to leptin in patients with osteoarthritis. Arthritis Res Ther 2010;12:R112.

4 Grundy SM: Obesity, metabolic syndrome, and cardiovascular disease. J Clin Endocrinol Metab 2004;89:25952600.

5 Grotle M, Hagen KB, Natvig B, Dahl FA, Kvien TK: Obesity and osteoarthritis in knee, hip, and/or hand: an epidemiological study in the general population with 10 years follow-up. BMC Musculoskelet Disord 2008;9: 132-136.

-6 Waldron HA: Association between osteoarthritis of the hand and knee in a population of skeletons from London. Ann Rheum Dis 1997;56:116-118.

7 Hart DJ, Spector TD: The relationship of obesity, fat distribution, and osteoarthritis in women in the general population: the Chingford Study. J Rheumatol 1993;20:331-335.

-8 Simopoulou T, Malizos KN, Iliopoulos D, Stefanou N, Papatheodorou L, Ioannou M, Tsezou A: Differential expression of leptin and leptin's receptor isoform (Ob-Rb) mRNA between advanced and minimally affected osteoarthritic cartilage; effect on cartilage metabolism. Osteoarthritis Cartilage 2007;15:872-883.

-9 Chen TH, Chen L, Hsieh MS, Chang CP, Chou DT, Tsai SH: Evidence for a protective role for adiponectin in osteoarthritis. Biochim Biophys Acta 2006;1762:711-718.

10 Lago R, Gomez R, Otero M, Lago F, Gallego R, Dieguez C, Gomez-Reino JJ, Gualillo O: A new player in cartilage homeostatsis: adiponectin induces nitric oxide synthase type II and pro-inflammatory cytokines in chondrocytes. Osteoarthritis Cartilage 2008;16:1101-1109.

11 Bokarewa M, Nagaev I, Dahlberg L, Smith U, Tarkowski A: Resistin, an adipokine with potent proinflammatory properties. J Immunol 2005;174:5789-5795.

12 Toussirot E, Streit G, Wendling D: The contribution of adipose tissue and adipokines to inflammation in joint diseases. Curr Med Chem 2007;14:1095-1100.

13 Gandhi R, Takahashi M, Smith N, Rizek R, Mahomed NN: The synovial fluid adiponectin-leptin ratio predicts pain with knee osteoarthritis. Clin Rheumatol 2010;29:1223-1228. 
Gandhi et al.: Obesity-Related Adipokines Predict Patient-Reported Shoulder Pain

14 Cibere JF, Baribaud F, Ma K, Sayre EC, Prestley N, Wong H, Thorne A, Singer J, Poole AR, Kopec JA, Guermazi A Nicolaou S, Esdaile JM: Serum biomarkers studies of symptomatic subjects with persistent knee pain, with and without $\mathrm{OA}$, reveal significant differences from asymptomatic subjects in systemic markers of inflammation suggesting differences in innate immunity. Osteo Cartilage 2010;18:S59.

15 Peterlin BL: The role of the adipocytokines adiponectin and leptin in migraine. J Am Osteopath Assoc 2009; 109:314-317.

16 Bedaiwy MA, Falcone T, Goldberg JM, Sharma RK, Nelson DR, Agarwal A: Peritoneal fluid leptin is associated with chronic pelvic pain but not infertility in endometriosis patients. Hum Reprod 2006;21:788-791.

17 Miranda H, Viikari-Juntura E, Martikainen R, Takala EP, Riihimaki H: Physical exercise and musculoskeletal pain among forest industry workers. Scand J Med Sci Sport 2001;11:239-246.

18 Viikari-Juntura E, Riihimaki H, Takala EP, Rauas S, Leppanen A, Malmivaara A: Factors predicting pain in the neck, shoulders and upper limbs in forestry work. People Work Res Rep 1993; 7:233-253.

19 Luime JJ, Kuiper JI, Koes BW, Verhaar JA, Miedema HS, Burdorf A: Work-related risk factors for the incidence and recurrence of shoulder and neck complaints among nursing-home and elderly care workers. Scand J Work Environ Health 2004;30:279-286.

20 Gandhi R, Takahashi M, Rizek R, Dessouki O, Mahomed NN: Obesity-related adipokines and shoulder osteoarthritis. J Rheumatol 2012;39:2046-2048.

21 Melzack R: The short-form McGill Pain Questionnaire. Pain 1987;30:191-197.

22 Gummesson C, Atroshi I, Ekdahl C: The disabilities of the arm, shoulder, and hand (DASH) outcome questionnaire: longitudinal construct validity and measuring self-rated health change after surgery. BMC Musculoskelet Disord 2003;4:11.

23 Cameron M, Briggs K, Steadman J: Reproducibility and reliability of the Outerbridge classification for grading chondral lesions of the knee arthroscopically. Am J Sports Med 2003;31:83-86.

24 Miller GD, Nicklas BJ, Davis CC, Ambrosius WT, Loeser RF, Messier SP: Is serum leptin related to physical function and is it modifiable through weight loss and exercise in older adults with knee osteoarthritis? Int J Obes Relat Metab Disord 2004;28:1383-1390.

25 Tian Y, Wang S, Ma Y, Lim G, Kim H, Mao J: Leptin enhances NMDA-induced spinal excitation in rats: a functional link between adipocytokine and neuropathic pain. Pain 2011;152:1263-1271.

-26 Hawker GA, Stewart L, French MR, Cibere J, Jordan JM, March L, Suarez-Almazor M, Gooberman-Hill R: Understanding the pain experience in hip and knee osteoarthritis - an OARSI/OMERACT initiative. Osteo Cartilage 2008;16:415-422.

-27 Dworkin RH, Corbin AE, Young JP Jr, Sharma U, LaMoreaux L, Bockbrader H, Garofalo EA, Poole RM: Pregabalin for the treatment of postherpetic neuralgia: a randomized, placebo-controlled trial. Neurology 2003;60:12741283.

-28 Rasmussen PV, Sindrup SH, Jensen TS, Bach FW: Symptoms and signs in patients with suspected neuropathic pain. Pain 2004;110:461-469.

-29 Gilron I, Bailey JM, Tu D, Holden RR, Weaver DF, Houlden RL: Morphine, gabapentin, or their combination for neuropathic pain. N Engl J Med 2005;352:1324-1334.

30 Tilg H, Moschen AR: Adipocytokines: mediators linking adipose tissue, inflammation and immunity. Nat Rev Immunol 2006;6:772-783.

-31 Ehling A, Schaffler A, Herfarth H, Tarner IH, Anders S, Distler O, Paul G, Distler J, Gay S, Schölmerich J, Neumann E, Müller-Ladner U: The potential of adiponectin in driving arthritis. J Immunol 2006;176:4468-4478.

32 Gomez R, Scotece M, Conde J, Gomez-Reino JJ, Lago F, Gualillo O: Adiponectin and leptin increase IL-8 production in human chondrocytes. Ann Rheum Dis 2011;70:2052-2054. 